\title{
Unsupervised Texture Segmentation using Active Contours and Local Distributions of Gaussian Markov Random Field Parameters
}

\author{
Chathurika Dharmagunawardhana ${ }^{1}$ \\ cd6g10@ecs.soton.ac.uk \\ Sasan Mahmoodi ${ }^{1}$ \\ sm3@ecs.soton.ac.uk \\ Michael Bennett ${ }^{2}$ \\ michael.bennett@soton.ac.uk \\ Mahesan Niranjan ${ }^{1}$ \\ mn@ecs.soton.ac.uk
}
${ }^{1}$ School of Electronics and Computer
Science, University of Southampton, Southampton, UK
${ }^{2}$ National Institute for Health Research, Southampton Respiratory Biomedical Research Unit, University Hospital Southampton NHS Foundation Trust, Tremona Road, Southampton, UK

\begin{abstract}
In this paper, local distributions of low order Gaussian Markov Random Field (GMRF) model parameters are proposed as texture features for unsupervised texture segmentation. Instead of using model parameters as texture features, we exploit the variations in parameter estimates found by model fitting in local region around the given pixel. The spatially localized estimation process is carried out by maximum likelihood method employing a moderately small estimation window which leads to modeling of partial texture characteristics belonging to the local region. Hence significant fluctuations occur in the estimates which can be related to texture pattern complexity. The variations occurred in estimates are quantified by normalized local histograms. Selection of an accurate window size for histogram calculation is crucial and is achieved by a technique based on the entropy of textures. These texture features expand the possibility of using relatively low order GMRF model parameters for segmenting fine to very large texture patterns and offer lower computational cost. Small estimation windows result in better boundary localization. Unsupervised segmentation is performed by integrated active contours, combining the region and boundary information. Experimental results on statistical and structural component textures show improved discriminative ability of the features compared to some recent algorithms in the literature.
\end{abstract}

\section{Introduction}

Texture segmentation has received considerable attention in recent computer vision research because texture features are one of the important elements in human understanding of visual data $[\square, \mathbf{Q}, \square]$. Preceding studies have demonstrated that statistical model based texture features yield good results in texture segmentation [日]. MRF theory provides a means of modeling context dependent patterns such as textures and object features [ $\square]$. Gaussian 
MRFs (GMRF) are a special version of MRFs. One of the early methods in GMRF literature along with unsupervised texture segmentation is introduced in [प]]. Other studies which employ these features are reported in $[\mathbf{\square}, \mathbf{\nabla}, \mathbb{\square}, \mathbb{\square}, \mathbb{\square}]$. In these studies estimated model parameters of GMRF have been used as an effective texture feature and known as the conventional or classical GMRF features (CGMRF) [ $[\mathbf{Q}, \mathbb{Z}]$. Successful attempts have been made in literature for harnessing improved GMRF based texture features. A step by step least square (SSLS) method is suggested in [ $[2]$ to estimate parameters according to a priority sequence. In [ $[\mathbf{D}]$ an improved estimation scheme is proposed to find estimators that can be applied in a non-stationary framework. A hierarchical GMRF for parameter estimation is proposed in [四], and a unified model of GMRF and mixtures of Gaussian for texture segmentation has been proposed in [四]. A new feature formulated based on estimated model parameters are suggested in [Q].

The present paper examines the use of local distributions of low order GMRF parameters, which are estimated via a localized estimation process which is restricted to a small estimation window size. They model the partial texture characteristics of the local region, hence the estimates inherit serious fluctuations over the spatial domain with a systematic pattern. It turns out that the variations on these inconsistent estimates surprisingly provide more discriminative features compared to consistent model parameters which are employed in CGMRF method. These spatial variations of biased estimates can be absorbed into normalized local histograms. Also smaller estimation windows help to maintain well localized boundaries. Incorrect model selection, for example to capture details of a very large texture pattern or a texture image with two very close textures, reduces the discriminative ability of the CGMRF features. Even though model selection is important, there exists a difficulty in employing large neighborhood sizes because the number of model parameters required to estimate, increases drastically by raising the size of the neighborhood. Yet the local distributions of estimates have the ability to successfully segment fine to very large texture patterns with a comparatively low order GMRF despite of texture pattern size. However, it is important to note that the accuracy of segmentation highly depends on the window size used to calculate histograms. Since variations occurred in the estimates have a relation to the texture pattern, the correct window size for this purpose is assumed to be nearly equal to texture pattern size. Therefore a technique to automate the selection of window size by considering entropy of a texture image is proposed.

The features are smoothed using diffusion via Beltrami flow and directed to the integrated active contour model [ $\square]$ ] for unsupervised texture segmentation. The procedure is unsupervised in the sense that it does not incorporate any training sample set. Instead of the level set based evolution scheme, shape characteristic function is employed as proposed in [D] to increase performance. The results reveal that the features proposed here produce superior texture segmentation performance.

The rest of the paper is organized as follows. The feature extraction method and the unsupervised segmentation method are discussed in section 2 and section 3 respectively. Section 4 presents the experimental results. Conclusions are drawn in section 5.

\section{Feature Extraction Method}

\subsection{Gaussian Markov Random Field Model}

Model parameters of the conditional distribution of GMRF offer a satisfactory feature set [Q]. 
Some main concepts of conditional GMRF models are briefly restated here. Markovianity denotes to the fact that a pixel value depends only on its neighboring pixel values. The GMRF model assumes that the intensity value of each pixel in the texture image is drawn from a Gaussian distribution function. Let $\Omega$ represents the set of grid points on a two dimensional image lattice. Then, $\Omega=\{(x, y) \mid 1 \leq x \leq$ Imwidth, $1 \leq y \leq$ Imheight $\}$, where Imwidth and Imheight are the width and height of the image. The intensity value of the pixel $s$ at $(x, y)$ position is given by $g_{s} \in[0,255]$ and $N_{s}$ denotes its neighborhood. Then the local conditional probability density function has the form,

$$
p\left(g_{s} \mid g_{s+r}, r \in N_{s}\right)=\frac{1}{\sqrt{2 \pi} \sigma} \exp \left\{-\frac{\left(g_{s}-\sum_{r \in N_{s}} \alpha_{s+r} g_{s+r}\right)^{2}}{2 \sigma^{2}}\right\}
$$

where $\alpha_{s+r}$ is the interaction parameter which measures the influence on $g_{s}$ by intensity value $g_{s+r}$ of a neighboring pixel at the neighbor location $r \in N_{s}$. Assume that pixels in symmetric positions about pixel $s$ have identical parameters. i.e $\alpha_{s+r}=\alpha_{s-r}$ with $r \in \tilde{N}_{s}$ where $\tilde{N}_{s}$, is the asymmetric neighbor set such that if $r \in \tilde{N}_{s}$, then $-r \notin \tilde{N}_{s}$ and $N_{s}=\left\{r \mid r \in \tilde{N}_{s}\right\} \cup\left\{r \mid-r \in \tilde{N}_{s}\right\}$. Note that by the symmetric criterion the number of interaction parameters are halved [ष्ठ].

The model parameters can then be found by maximum likelihood estimation (MLE). The MLE estimates of model parameters corresponding to the pixel at $s$ is given by,

$$
\begin{gathered}
\alpha=\left[\sum_{j=1}^{N} \mathbf{q}_{j} \mathbf{q}_{j}^{T}\right]^{-1}\left[\sum_{j=1}^{N} \mathbf{q}_{j} g_{s_{j}}\right] \\
\sigma^{2}=\frac{1}{N} \sum_{j=1}^{N}\left(g_{s_{j}}-\mathbf{q}_{j}^{T} \alpha\right)^{2}
\end{gathered}
$$

where $\alpha=\operatorname{col}\left[\alpha_{s+r} \mid r \in \tilde{N}_{s}\right]$ and $\mathbf{q}_{j}=\operatorname{col}\left[G_{s_{j}+r} \mid r \in \tilde{N}_{s_{j}}\right]$. For each pixel a square estimation window of size $p$, pre-processed to have zero mean, centered at the pixel is considered in order to formulate texture features. $\mathrm{N}$ overlapping square neighborhoods of size $n$ are extracted from the estimation window, which is then used as different realizations to estimate parameters from equations (2) and (3) (Note that for GMRF model, it can be shown that MLE and least square estimation lead to the same set of equations [四]). Square neighborhoods are employed here, because model selection is not a prior concern when using local distributions as describes in section 2.2. At the boundary of the image, symmetric boundary condition is assumed.

\subsection{GMRF Local Distributions Based Texture Features}

An important property of a reliable texture feature is that it obtains minimal intraclass variations [ []$]$. Then, considering CGMRF features, the estimated values corresponding to a specific model parameter should attain reduced intraclass variations i.e. their values should be uniform as much as possible over the region of one texture and give a near piece-wise constant sense to the feature image comprising more than one texture.

It is known that a model parameter of GMRF directly represent interaction between a pixel and a pixel located in its neighborhood [ㅁ, $\square]$. By observing the different neighborhoods appearing on a certain texture, it is reasonable to assume that values of a specific model parameter should allow some variations depending on complexity of the texture 
pattern, rather than assuming a uniform dispersion. Therefore, estimates of model parameters should follow a certain distribution of values [四]. These variations in estimates are smoothed out by the consistent estimation process which uses sufficiently large estimation window, contributing towards an information loss regarding distributions of model parameter values. Hence in this paper we examine variations occurring in estimates of GMRF parameters, which are estimated via a spatially restricted estimation process using a smaller estimation window. A small estimation window provides a small number of local samples for the estimation process. Therefore it does not fit the global texture model as necessary but over-fit into given local samples providing a partial texture model describing a local region of the texture. These biased estimates inherit serious fluctuations over the spatial domain with a systematic pattern related to re-occurrences of local regions of the texture. These spatial variations of biased estimates are assumed to represent the variations occur in model parameters and can be quantified by local normalized histograms. Also exploiting smaller estimation windows lead to well localized boundaries.

Local distributions of biased estimates also represent some degree of robustness to the choice of neighborhood size. Estimates achieved by involving a wrong neighborhood size does not represent the true texture model [ष]. In the literature of GMRF, it is noticed that neighborhood size is restricted to small values, maintaining less computational cost [ $\mathbf{Q}, \mathbf{Q}, \mathbf{Q}$, [ם, 四]. When the neighborhood size is restricted, the estimates will deviate from true values causing monotonic shifting of the numerical value obtained for uniform consistent estimates. These monotonic shifts cause problems, specially in situations where close component textures are to be segmented. Yet the fluctuation patterns occurring in biased estimates retain their significance for representing a texture, despite the restriction posed on neighborhood size. Hence segmentation of two textures with close characteristics could not be successful with direct use of consistent estimates although local distributions of bias estimates can produce successful results. Therefore proposed features can segment fine to very large texture patterns with a comparatively small neighborhood size with respect to pattern size. Hence the selection of model is not a prime concern anymore.

Estimation of model parameters results in transformed images of model parameter estimates (feature images). Each transformed image represents estimated values of a certain model parameter for all the pixels in the image. For every pixel $s$ at $(x, y)$ on a transformed image, a square window of size $b$ centered at that pixel is used to calculate the local normalized histogram. These local distributions of model parameter values are here after referred to as the parameter-local histogram (PL histogram).

Selection of an accurate window size $b$ is very important for a better segmentation. The window size chosen should be able to capture the actual pattern size of the texture. In [W] a feature deviation function (FDF) has been employed to find minimum intra-class variation or feature deviation to construct the texture features. Here instead of FDF, Shannon's entropy is used because our purpose is to measure the randomness of a texture. Let $H(b)$ represent the average entropy of all non overlapping windows of size $b$ on a texture image. Then the normalized derivative of $H(b)$ is considered as,

$$
H^{*}(b)=\frac{|H(b)-H(b-1)|}{H_{\min }}
$$

where $H_{\min }$ is the minimum value of average entropy function $\mathrm{H}(\mathrm{b})$. It is observed that fine texture patterns have a entropy function with higher values and steeper slopes compared to larger texture patterns (Figure (1-b)). This is because fine textures contain more information with smaller $b$ values and hence higher entropy values. Also they reach the saturation 


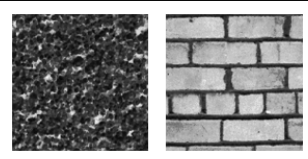

(a)

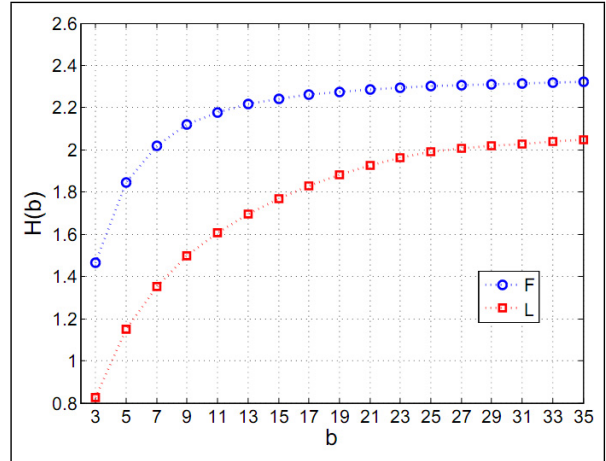

(b)

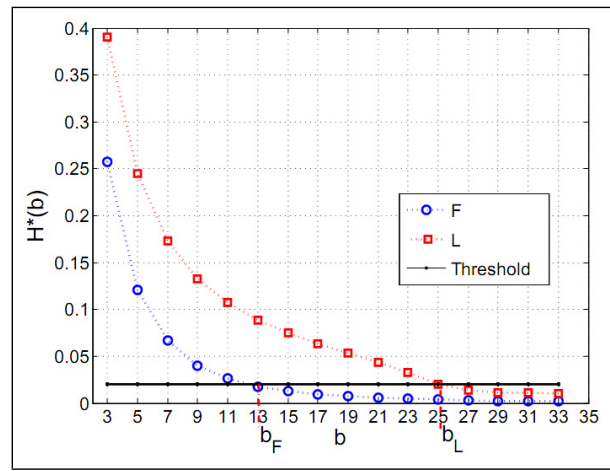

(c)

Figure 1: (a) Two textures characterized with fine $(\mathrm{F})$ and large $(\mathrm{L})$ texture patterns, (b) average entropy function, (c) normalized derivative of entropy

state quickly because the degree of randomness of the pattern is limited to a smaller window size. Therefore we use equation (4) which captures both aspects. To find an accurate value of $b$, a threshold is found experimentally. In our experiments, the images are comprised of two textures and used in the above entropy method to calculate $b$ with Shannon entropy. Therefore the resulting average entropy curve associates the characteristics of both texture entropy functions and also depends on percentage of occupancy of each texture and shapes of the texture regions in the image. The value obtained for $b$ here, is a suitable average window size for both textures rather than for individual textures. It is noted that both the slope and the $H_{\text {min }}$ which are now influenced by both textures, are important to find the correct value for $b$. The information from slope provides a crude value for $b$ and the information from $H_{\text {min }}$ further refines it and drives it towards the correct value of $b$. The obtained window size $b$ in this way is sufficient to capture local spatial variations of estimated parameters of both the textures.

Once the accurate window size $b$ is found, PL histograms are calculated as follows. On the transformed image $\mathbf{I}^{k}$, of the $k^{\text {th }}$ model parameter, at a certain pixel $s$ located at $(x, y)$ the PL histogram formulated using a patch $\mathbf{I}_{b \times b @(x, y)}^{k}$ of size $b \times b$ around $(x, y)$ is given by,

$$
\mathbf{F}_{s}^{k}(z)=\frac{1}{b^{2}} \sum_{u} \delta\left(z-\mathbf{I}_{b \times b @(s)}^{k}(u)\right)
$$

where $u$ is the linear index of the pixel location on the patch and $\delta$ is the Dirac delta function. If the mean and standard deviation of parameter values on $\mathbf{I}^{k}$ is given by $\mu^{k}$ and $s t d^{k}$, then the range of the histogram values $z$ is chosen to be $\mu^{k}-3 s t d^{k} \leq z \leq \mu^{k}+3 s t d^{k}$. Number of bins employed is always fixed. The full feature matrix of a pixel $s$ at $(x, y)$, corresponding to all model parameters, is the concatenated PL histograms at $(x, y)$ which can be formulated as,

$$
\mathbf{F}_{s}=\left(\mathbf{F}_{s}^{1}, \mathbf{F}_{s}^{2}, \ldots, \mathbf{F}_{s}^{k}, \ldots, \mathbf{F}_{s}^{\left(n^{2}+1\right) / 2}\right)
$$


or it can be concatenated into a vector rather than a matrix as above. To reduce the amount of noise present in the PL histogram features and smooth the features, a diffusion technique via Beltrami flow, comprehensively explained in Sagiv et al. [ $\square$ ] is employed. This non linear smoothing scheme is chosen because it has the ability to preserve the edges compared to linear diffusion such as Gaussian filtering, hence it is very useful in the present application which tries to find the boundaries between two textures. Each bin image, created by the values pertaining to all the image pixels with respect to a certain bin of PL histogram, is iteratively smoothed.

\section{Unsupervised Segmentation Model}

An integrated framework which combines boundary and region information in active contour modality presented by Sagiv et al. [ $\square]$ is used as the segmentation method. Active contours is previously combined with GMRF model in []ㅣ. The segmentation method discussed in $[\square]$ is briefly explained here from the view point of PL histogram features.

Let $C$ be the evolving contour, and $\mathbf{F}_{\text {in }}^{k}$ and $\mathbf{F}_{\text {out }}^{k}$ be the average PL histogram of inside and outside the contour $C$. Then the energy functional to be minimized is,

$$
\begin{aligned}
E\left(\mathbf{F}_{\text {in }}^{k}, \mathbf{F}_{\text {out }}^{k}, C\right)= & \mu \int_{\text {on }(C)} h(x, y) \mathrm{d} x \mathrm{~d} y+\lambda_{\text {in }} \sum_{k=1}^{\left(n^{2}+1\right) / 2} \int_{\text {inside }(C)} D\left(\mathbf{F}_{s}^{k}, \mathbf{F}_{\text {in }}^{k}\right) \mathrm{d} x \mathrm{~d} y \\
& +\lambda_{\text {out }} \sum_{k=1}^{\left(n^{2}+1\right) / 2} \int_{\text {outside }(C)} D\left(\mathbf{F}_{s}^{k}, \mathbf{F}_{\text {out }}^{k}\right) \mathrm{d} x \mathrm{~d} y
\end{aligned}
$$

where $D(.,$.$) denotes the L1 norm distance between PL histogram features at a pixel$ and the corresponding average PL histogram. $\mu, \lambda_{\text {in }}$ and $\lambda_{\text {out }}$ are constants which determine the contribution of each term to the total energy. The functional in equation (7) assumes the texture image consists of two textures. Functional 7 can be generalized for images with more than two textures by adapting a multi-phase scheme similar to the method proposed in [四]. $h$ is the inverse edge image, where edges, or more meaningfully the boundaries between two texture regions, are represented as lower intensity values. $\mathbf{F}_{\text {in }}^{k}$ and $\mathbf{F}_{\text {out }}^{k}$ are calculated iteratively, as the average PL histograms of inside and outside of the evolving contour.

The $h$ is calculated via the metric of the 2D image manifold [ $\square]$ ]. Let the $i^{t h}$ bin image is represented by $\mathbf{W}^{i}=\left[W^{i}(x, y) \mid 1 \leq x \leq\right.$ Imwidth, $1 \leq y \leq$ Imheight $]$. Then the metric of the feature space is given by,

$$
g(x, y)=\left(\begin{array}{cc}
1+\sum_{i=1}^{L}\left(W_{x}^{i}\right)^{2} & \sum_{i=1}^{L} W_{x}^{i} W_{y}^{i} \\
\sum_{i=1}^{L} W_{x}^{i} W_{y}^{i} & 1+\sum_{i=1}^{L}\left(W_{y}^{i}\right)^{2}
\end{array}\right)
$$

where $W_{x}^{i}$ and $W_{y}^{i}$ are partial derivatives w.r.t. $x$ and $y$. Partial derivatives can be obtained by finite difference method applied on to $i^{t h}$ bin image and $\mathrm{L}$ is equal to the number of total bins in the vector form concatenated PL histogram.

Since the metric $g$ is associated with measuring distances on manifolds, its components represent the rate of change of a given manifold in a specific direction. Hence the determinant 
of $g$ has higher values when a strong gradient presents [ $\square$ ]]. Therefore metric $g$ can be used for edge detection. Then the value $h(x, y)$ at $(x, y)$ of inverse edge image $h$ can be formed as,

$$
h(x, y)=\frac{1}{\operatorname{det}(g(x, y))}
$$

where $\operatorname{det}()$ represents taking determinant of a matrix and $g(x, y)$ represent matrix of $g$ metric for the location $(x, y)$. In energy functional (7), the first term is associated with the boundary information while the second and third terms are responsible for regional information. The evolution equation obtained by minimizing energy functional (7) is given by,

$$
\frac{\partial \chi}{\partial t}=\mu \operatorname{div}\left(h \frac{\nabla \chi}{|\nabla \chi|}\right)-\lambda_{\text {in }} \sum_{k=1}^{\left(n^{2}+1\right) / 2} D\left(\mathbf{F}^{k}, \mathbf{F}_{\text {in }}^{k}\right)+\lambda_{\text {out }} \sum_{k=1}^{\left(n^{2}+1\right) / 2} D\left(\mathbf{F}^{k}, \mathbf{F}_{\text {out }}^{k}\right)
$$

Here instead of a level set formulation using the sign distance function $\phi(x, y)$ [四], $\chi(x, y)$, the shape characteristic function is used, where $H(\phi(x, y))=\chi(x, y)[\square](H$ is the Heaviside function). The shape-based active contour method to construct the evolution function has a faster convergence, less memory usage and better performance in the presence of noise. To obtain the discrete version of equation (10) we use the semi implicit finite difference method discussed in [四].

\section{Experimental Results}

In order to demonstrate the performance of the proposed texture segmentation method, a variety of texture images created from Brodatz textures $[\square]$ are employed. The selected texture images include fine to very large texture patterns. The texture regions are comprised of convex shapes, internal contours and disconnected regions. In some cases, textures are very similar to each other in their texture characteristics, giving hardly noticeable region boundaries to the human eye. In the feature extraction phase, 10 bins for PL histogram and $p=n+2$ are chosen. $p$ is the size of the estimation window to extract sample neighborhoods and it is relatively small, hence spatially localizing the estimation process. $p=n+16$ was used for CGMRF to obtain a consistent estimation [ख]]. The neighborhood size; $n$ is fixed to $n=5$ which is a relatively small order. The value for $b$ used in histogram calculation is found by the entropy scheme proposed here, with a threshold value of 0.02 which is obtained experimentally. In the smoothing phase, Beltrami diffusion is applied to each bin image separately for 20 iterations with a 0.1 time step. In the segmentation phase, the smoothing parameter $\varepsilon$ is chosen to be 3 and the size of the smoothing filter is set to $19 \times 19 \mathrm{pxls}$. We also let $\lambda_{\text {in }}=\lambda_{\text {out }}=\lambda_{i}$.

The texture segmentation results on statistical and structural texture patterns with fine to very large patterns are illustrated in figure (2). For the result in the figures $(2-e, f, g), \mathrm{n}=$ 5 is not a sufficient neighborhood size to capture the texture pattern. The consequences of using insufficient neighborhood sizes are alleviated by using the PL histogram as presented in figures $(2-i, j, k)$. The percentage error rate of incorrectly segmented pixels with respect to the total number of pixels in the image $\left(s_{e}\right)$ is given below each segmented image.

Texture segmentation method proposed here is evaluated against Sagiv et al. Gabor based features $[\square]$ (Figure (3)). Textures in figure (3-a) and (3-e) are quite challenging for human vision to detect. The difficulty in the Gabor feature based method is to select a suitable subset of filters. A subset of fine tuned filters is manually selected for the statistical textures 


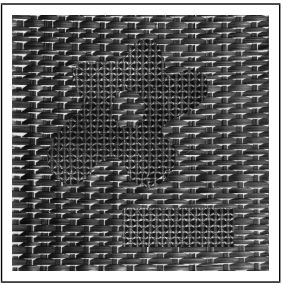

(a)

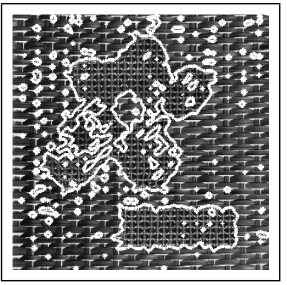

(e) $s_{e}=10.8 \%$

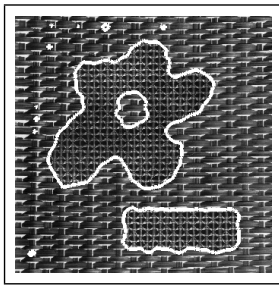

(i) $s_{e}=1.68 \%$

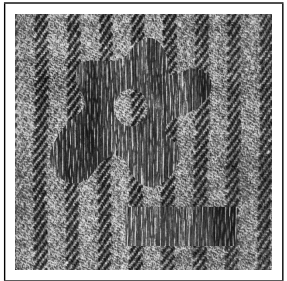

(b)

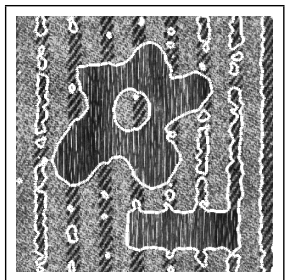

(f) $s_{e}=14.6 \%$

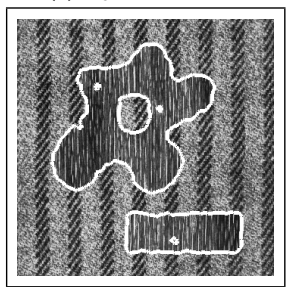

(j) $s_{e}=1.09 \%$

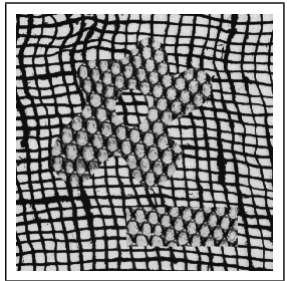

(c)

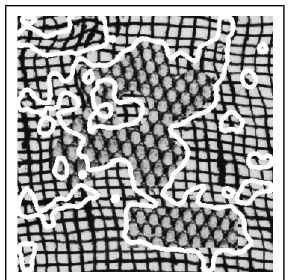

(g) $s_{e}=22.6 \%$

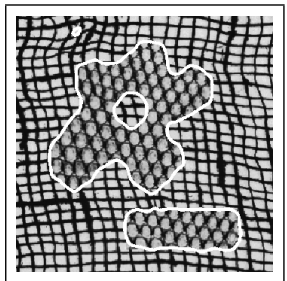

(k) $s_{e}=2.54 \%$

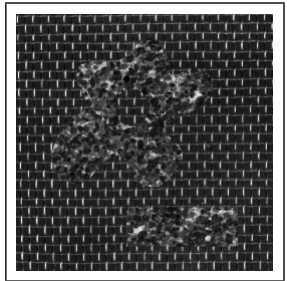

(d)

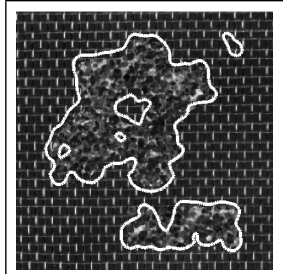

(h) $s_{e}=7.16 \%$

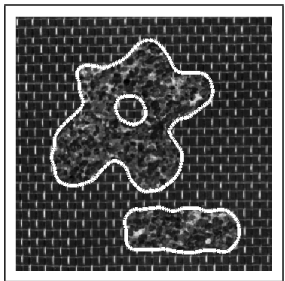

(1) $s_{e}=2.90 \%$

Figure 2: Segmentation Results. (a)-(d) the original images, (a) and (b) are $640 \times 640$ and (c) and (d) are $256 \times 256$, (e)- (h) using CGMRF[ $[$, 口] and (i)-(l) using PL histogram. $\mu=10^{-7}$ and $\lambda_{i}=20$.

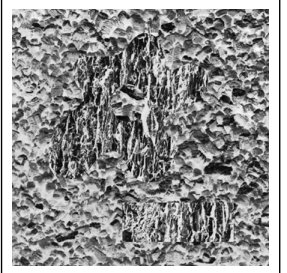

(a)

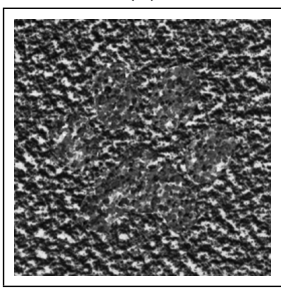

(e)

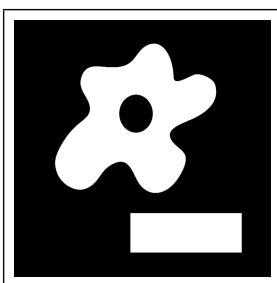

(b)

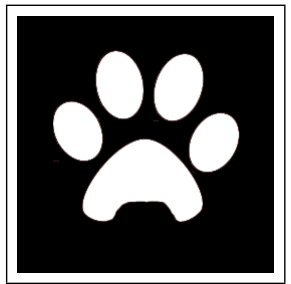

(f)

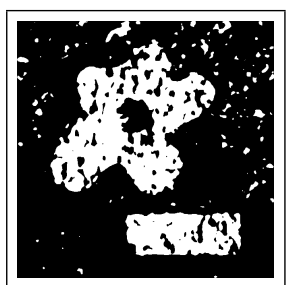

(c) $s_{e}=8.34 \%$

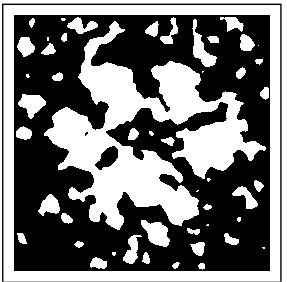

(g) $s_{e}=17.9 \%$

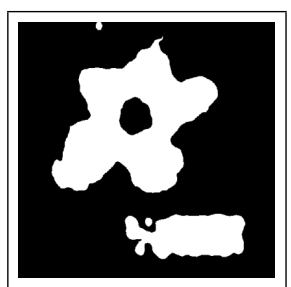

(d) $s_{e}=2.70 \%$

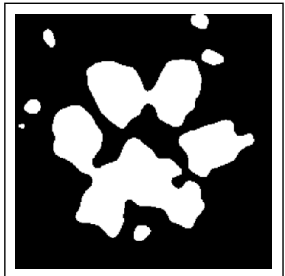

(h) $s_{e}=7.03 \%$

Figure 3: Statistical texture segmentation results. (a), (e) original images (a) is $640 \times$ 640 and (e) is $256 \times 256$, (b), (f) segmentation target, (c), (g) using Gabor features and (d), (h) using PL histograms. Filters used, in (c) $f=[0.15,0.3,0.35,0.4,0.45]$, in (g) $f=$ $[0.15,0.3,0.35,0.4]$. \# of $\theta=6, \mu=10^{-7}$ and $\lambda_{i}=20$. 


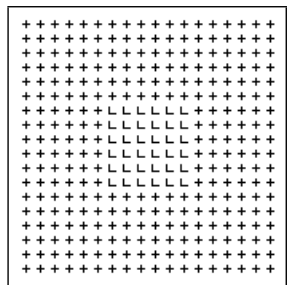

(a)

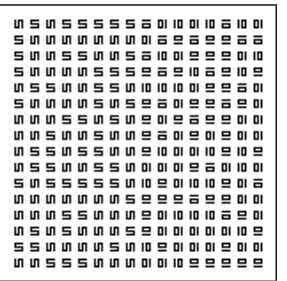

(e)

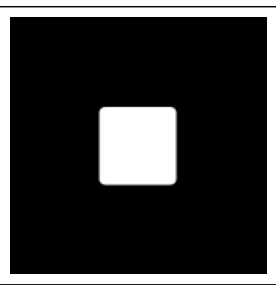

(b)

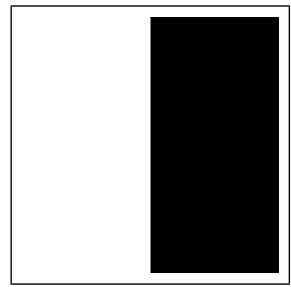

(f)

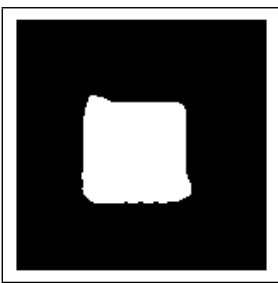

(c) $s_{e}=4.17 \%$

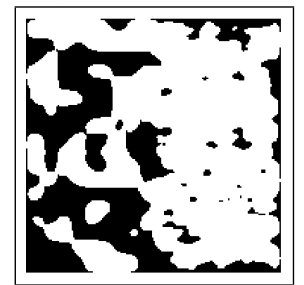

(g) $s_{e}=32.6 \%$

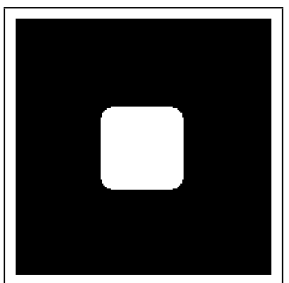

(d) $s_{e}=0.63 \%$

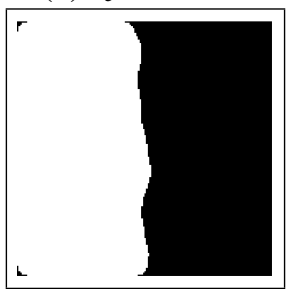

(h) $s_{e}=1.64 \%$

Figure 4: Segmentation of texture images involved in the psychophysical studies of textures. (a), (e) original images. (b) and (f) segmentation target. (c) and (g) segmentation results using Gabor features, (d) and (h) segmentation results using the method proposed here. Images are $180 \times 180$.

of figure (3) to favor the filter based segmentation method in [ $\square]$. Our segmentation method proposed here produces more accurate results in segmenting the challenging images of figure (3) consisting of two statistical textures, compared to the method proposed in [ $\square$ ].

Finally, texture images used in psychophysical studies are employed here to analyze the segmentation performance of the PL histogram. Figure (4- $a$ ) has two textures with close power spectra and figure (4-e) has two textures with identical second order statistics [ $[$ ]. As observed from figure (4-e) the texture is even difficult for human perception to segment. The images are recreated from [ $[$ ] . The segmentation based on PL histogram perform well compared to Gabor feature based method in [प]]. Segmentation result in figure (4- $h$ ) is achieved with $b=21$. When $b$ is increased manually it starts to recognize some similarity in the placement of rotated versions of texture elements within a texture and achieve a better segmentation.

\section{Conclusions}

In this study the use of local distributions of biased estimates of low order GMRF model parameters as a texture feature in an unsupervised texture segmentation framework is explored. The texture features proposed here, the PL histograms, (1) are more robust against the deviation of estimates from true model parameter value as a result of improper model selection, (2) take advantage of variations in inconsistent estimates, (3) achieve well localized boundaries, (4) extend successful segmentation of fine to very large texture patterns with smaller neighborhoods and (5) can easily be formulated by local histograms, maintaining simplicity and efficiency. The performance of the proposed texture features on other texture databases should be evaluated in future. The limitations of using an ad hoc entropy based method for window size selection of histogram calculation has to be analyzed further and improved. The integrated active contour model employed here is suitable for a two-phase segmentation and 
therefore, the test images consist of two textures. A multi-phase active contour model, can be exploited for images containing more than two textures. Finally our segmentation algorithm can successfully segment challenging homogeneous statistical and structural textures.

\section{References}

[1] J. Amelung and K. Vogel. Automated window size determination for texture defect detection. In Proc. British Machine Vision Conference, pages 105-114, 1994.

[2] P. Brodatz. Textures: A Photographic Album for Artists and Designers. New York: Dover, ISBN: 0486216691, 1996.

[3] E. Cesmeli and D. Wang. Texture segmentation using Gaussian- Markov random fields and neural oscillator networks. IEEE transactions on Neural Networks, 12(2):394-404, 2001.

[4] R. Chellappa and S. Chatterjee. Classification of textures using Gaussian Markov random fields. IEEE Transactions on Acoustics Speech and Signal Processing, 33(4): 959-963, 1985.

[5] C. H. Chen, L. F. Pau, and P. S. P. Wang. Handbook of Pattern Recognition and Computer Vision. World Scientific Publishing Co. Pte Ltd, ISBN: 9810211368, 1993.

[6] H. Deng and D. A. Clausi. Gaussian Markov random field rotation-invariant features for image classification. IEEE transactions on pattern analysis and machine intelligence, 26(7):951-5, 2004.

[7] X. Descombes, M. Sigelle, and F. Preteux. Estimating Gaussian Markov random field parameters in a non stationary framework: Application to remote sensing imaging. IEEE transactions on image processing, 8(4):490-503, 1999.

[8] X. Huang, J. Dong, and M. Wang. Paper web defection segmentation using GaussMarkov random field texture features. In Proc. IEEE Int'l Conf. Image Analysis and Signal Processing, pages 167-170, 2011.

[9] A. K. Jain and F. Farrokhnia. Unsupervised texture segmentation using Gabor filters. In Proc. IEEE Int'l Conf. Systems Man and Cybernetics, pages 14-19, 1990.

[10] D. H. Kim, D. Yun, and S. U. Lee. New MRF parameter estimation technique for texture image segmentation using hierarchical GMRF model based on random spatial interaction and mean field theory. In Proc. 18th Int'l Conf. Pattern Recognition, pages 365-368, 2006.

[11] S. Z. Li. Markov Random Field Modeling in Image Analysis. Springer-Verlag London Ltd, ISBN: 9781848002784, 2009.

[12] S. Mahmoodi. Shape-based active contours for fast video segmentation. IEEE Signal Processing Letters, 16(10):857-860, 2009.

[13] S. Mahmoodi and S. Gunn. Snake based unsupervised texture segmentation using Gaussian Markov random field models. In Proc. 18th IEEE Int'l Conf. Image Processing, pages 1-4, 2011. 
[14] B. S. Manjunath and R. Chellappa. Unsupervised texture segmentation using Markov random field models. IEEE transactions on pattern analysis and machine intelligence, 13(5):478-482, 1991.

[15] U. Peng, T. X. Wei, and F. J. Fu. A unified model of GMRF and MOG for image segmentation. In Proc. IEEE Int'l Conf. Image Processing, pages 1140-1143, 2005.

[16] M. Petrou and P. G. Sevilla. Image Processing, Dealing with Texture. John Wiley Sons Ltd, ISBN: 0470026286, 2006.

[17] C. Sagiv, N. A. Sochen, and Y. Zeevi. Integrated active contours for texture segmentation. IEEE transactions on image processing, 15(6):1633-46, 2006.

[18] S. Stan, G. Palubinskas, and M. Datcu. Bayesian selection of the neighbourhood order for Gauss-Markov texture models. Pattern recognition letters, 23:1229-1238, 2002.

[19] L. Vese and T. Chan. Active contours without edges. IEEE transactions on image processing, 10(2):266-77, 2001.

[20] L. Vese and T. Chan. A multiphase level set framework for image segmentation using the Mumford and Shah model. International Journal of Computer Vision, 50(3):271293, 2002.

[21] G. Xia, C. He, and H. Sun. Urban extraction from SAR images using local statistical characteristics and Gaussian Markov random field model. In Proc. 8th IEEE Int'l Conf. Signal Processing, pages 20-23, 2006.

[22] Y. Zhao, L. Zhang, P. Li, and B. Huang. Classification of high spatial resolution imagery using improved Gaussian Markov random-field-based texture features. IEEE Transactions on Geoscience and Remote Sensing, 45(5):1458-1468, 2007. 